\title{
Optical Remote Dimensional Inspection of Live Contact Wire in Train's Electro-Supply Network
}

\author{
Yu.V. Chugui ${ }^{1,2}$, A.G. Verkhogliad ${ }^{1}$, V.S. Bazin ${ }^{1}$, S.V. Kalichkin ${ }^{1}$, \\ V.E. Kalikin ${ }^{1,2}$, S.N. Makarov ${ }^{1}$, I.A. Vykhristyuk ${ }^{1}$ \\ ${ }^{1}$ Technological Design Institute of Scientific Instrument Engineering, \\ Siberian Branch of the Russian Academy of Sciences, 41, Russkaya str., Novosibirsk, 630058, Russia \\ 2 Novosibirsk State University, 2, Pirogova str., Novosibirsk, 630090, Russia \\ Email: chugui@tdisie.nsc.ru
}

\begin{abstract}
An optical structured illumination of $3 D$ object as a light band method for automatic noncontact wear measurement and defects detection of a contact wire network is presented. The experimental device can extract main technological wire parameters like remaining height with RMS value down to $0.15 \mathrm{~mm}$. Also the cross-sectional area of the wire can be measured with RMS area noise value of $1.5 \mathbf{~ m m}^{2}$, which is sufficient for a confident decision-making related to the automatic wire wears and defects on railways, tram and trolleybus lines, and similar systems of vehicles electro-supply.
\end{abstract}

Keywords: structured light method, non-contact remote inspection, profiling, shape measurement, contact wire.

\section{INTRODUCTION}

$\mathrm{R}$ AILROAD safety requires continuous dimensional inspection of geometrical parameters of wearing parts. One of the actual railroad tasks is inspection of a train's electro-supplying contact wire deterioration. During long operation periods of wire in use the defects take place and the wire surface wears out. As a result, the configuration of the wire is changed and it has to be measured.

The measurement method has to be noncontact since the wire is always under the live voltage up to several tens of $\mathrm{kV}$. The developed system has to be compact in size so as to allow its easy transportation. Due to the requirement of many wire cross-section measurements with small spacing step (less than $10 \mathrm{~mm}$ ), the method has to provide the corresponding resolution along the train's wire. The system has to be designed so as to allow the measurements of the remaining height of the wire with the error no more than 0.2 $\mathrm{mm}$ while the train is moving.

As it is known, the optical shadow method has been used [1-2] in the resolution of many industrial tasks. However, the shadow method has its own limitations. While obtaining the shadows from opaque concave objects, the geometry information is lost. Therefore such method is normally applied only for the opaque objects with convex shapes. The second important disadvantage of this method is that it poses certain limitations to the relative spatial arrangement of the light source and shadow image receiving plane: the source and receiver have to be located on the opposite sides of the object under geometrical inspection. As regards the train's wire geometrical inspection problem, the shadow method cannot be utilized in a practical way. That would cause very bulky system design and exclude the possibility of the wire surface defects inspection.

This article describes the structured light method for wire geometry measurement and defects inspection [2]. After the measurement method, the structural scheme of the measuring module, the image processing algorithm and the experimental testing results of industrial prototype of the developed system in real railway conditions are presented. The method can be applied to the different types of the contact wire which can be used in trains, trams, trolleybuses and other moving sliding wire electro-supplied vehicles.

\section{THE MEASUREMENT METHOD}

During long operation the contact wire can be subjected to some geometrical defects that require their timely detection and removal. Unworn-out contact wire (Fig.1a) and possible geometrical defects are shown in Fig.1 b, c, d. The main defect that leads to the increase of specific resistance and growth of contact wire break possibility is the decrease of the area of its cross-section because of continuous contact with collector (Fig.1b). The wire can also be turned in cross-section plane (Fig.1c) and made very thin in upturned position. The tension irregularity as well as some micro defects in wire can lead to the defect of "neck" type (Fig.1d), when the wire section simultaneously decreases along all directions. The contact wire wear is described by two parameters (Fig.1b), namely: width of wear $\mathrm{W}$ and wear depth $\mathrm{D}$, i.e. by the distance from the surface of unworn-out wire up to the contact surface of the worn-out one.

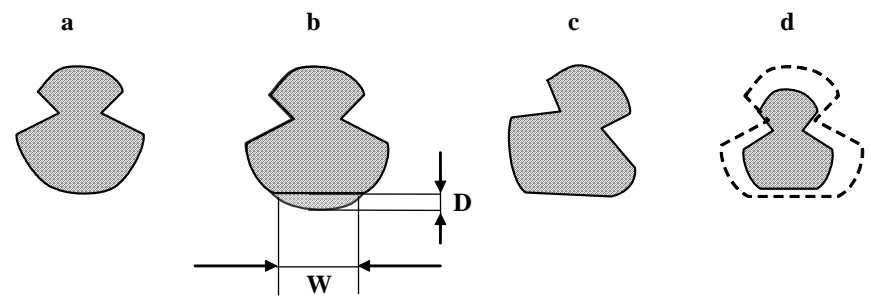

Fig.1 New contact wire cross-section (a) and possible contact wire defects: standard worn-out wire (b), "rotated wire" defect (c), "neck wire" defect (d)

For an automatic noncontact detection of the above mentioned defects as well as for the measurement of wear 
parameters, we have proposed the use of a structured light method [2]. The principle of this method is illustrated by Fig. 2. A special laser illuminator generates one or several transversal plane-shaped beams ("light knives"), which incident on the wire surface at some angles to its axis. The scattered light is captured by a video-camera from the other direction. The captured image represents the shape of the curve, which is formed by intersection of the plane-shaped beam with the curved wire surface.

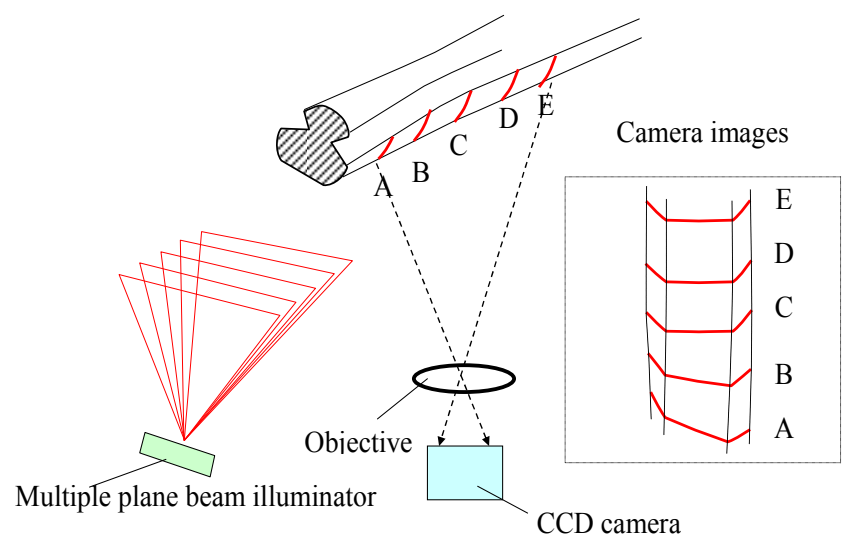

Fig.2 The structured light method for cross-section wire parameters measurement

As an example, the actual image of the wire, obtained by experimental system in the laboratory conditions, and calculated image parameters (see Fig. 1) are shown in Fig. 3. The dark curve demonstrates the predicted wire section profile obtained by image processing of the light crescent (bright arc-type shape). The crescent is formed as a result of the wire surface intersection by laser beam plane.

\section{THE MEASURING SYSTEM}

Using this method we have developed an industrial system prototype. The measuring system design is constrained by additional requirements, which depend on the design of the train's electro-supply network. For the train contact wire, the measurement field has the following dimensions: $\sim 1000 \mathrm{~mm}$ in width (since the wire can move in horizontal plane) and $\sim 1500 \mathrm{~mm}$ in depth (due to the possible vertical movements of wire relative to the rails). For such size of the measurement zone it is practically impossible to make such illumination distribution. Therefore, the solution of the problem was based on the tracking out of the wire's height by moving the laser illuminator and keeping the distance between this source and the wire constant. Moreover, in order to meet the requirements to the optical focal depth of the camera lens, the camera is moved relative to the wire in order to keep constant distance between the camera and wire equal to $2500 \mathrm{~mm}$. a)

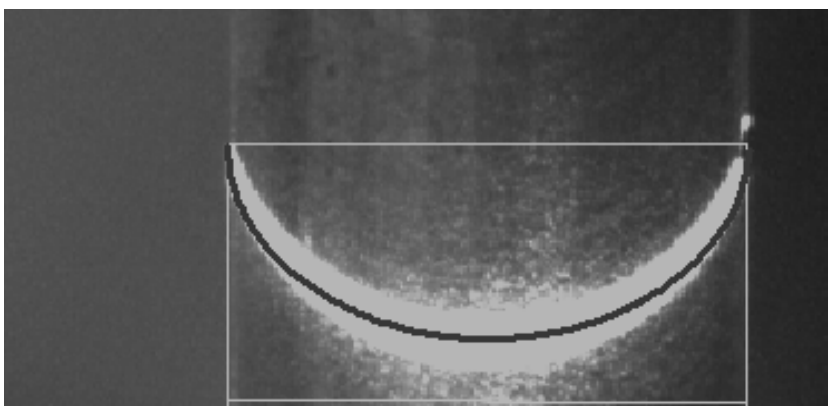

b)

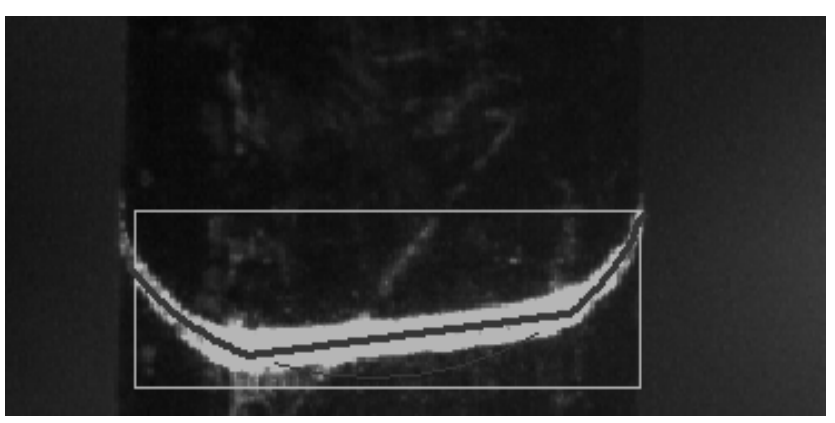

Fig.3 The contact wire cross-section image obtained under the laboratory experiments: unworn-out wire with the wear contact surface width $\mathrm{W}=1.1 \mathrm{~mm}(\mathrm{D}=0.0 \mathrm{~mm})(\mathrm{a})$; worn-out wire with wear parameters $\mathrm{W}=8.0 \mathrm{~mm}$ and $\mathrm{D}=1.4 \mathrm{~mm}(\mathrm{~b})$

Figure 4 demonstrates the structural scheme of the measuring system. Optical subsystem has a moving laser and two mirrors: one of them is fixed statically and other is moveable in order to adjust illumination. The tracking subsystem consists of a motor and controlling software application executed on $\mathrm{PC} 104+$ microcomputer. Three similar cameras were evenly spaced in order to extend the field of view in the horizontal plane. The developed measuring module, installed on the roof of an inspection car, and tested on "Seyatel" railroad station, is presented in Figure 5.

\section{THE IMAGE PROCESSING ALGORITHM}

The development of a real, practically working system takes the solution of some problems (tasks). One of such problems is an insufficient special resolution of an observing camera. The second problem that determines the measurement precision lies in the impossibility of beam focusing into a very thin line at the distance of about 2 meters. So, the total analysis of the video image is divided into some stages. 


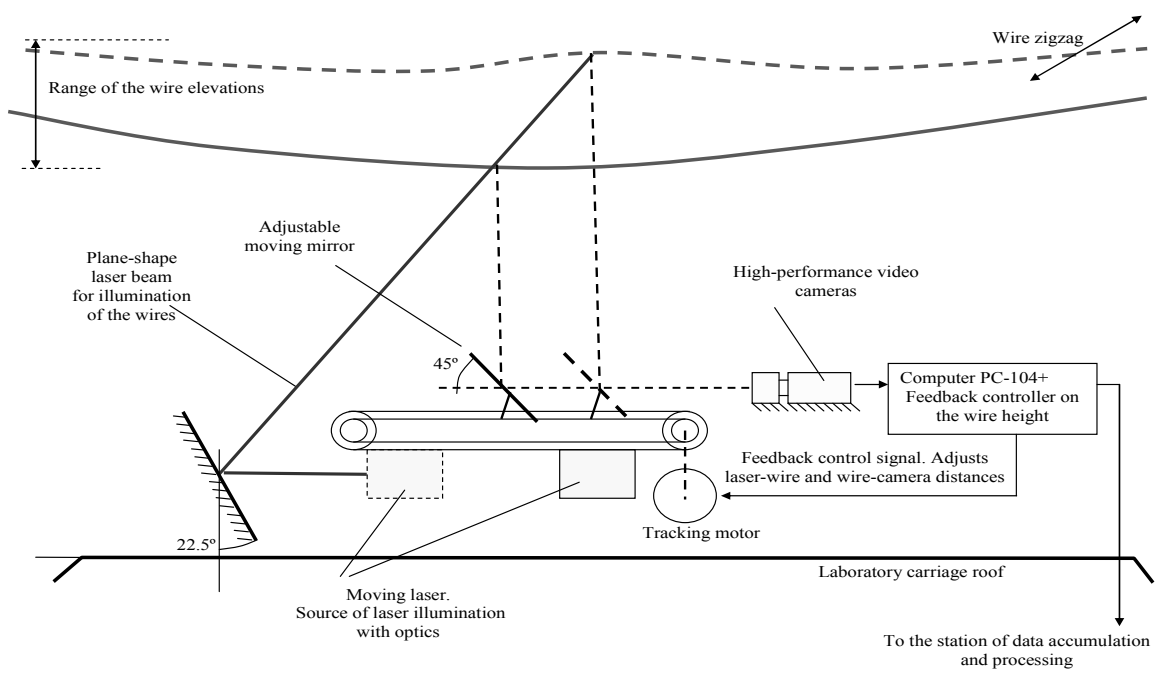

Fig.4 The structural scheme of the measuring system

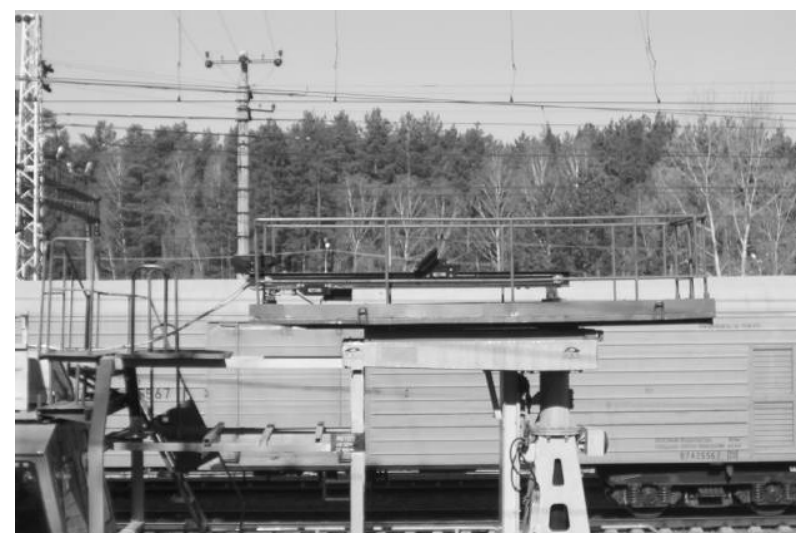

Fig.5 Measuring module installed on the roof of railway inspection car

The first step for image analysis is the search for video image informative sections, i.e. frame sections that contain the image of the wire dissection by laser beam. Such sections, which we will call "crescents" thereinafter, can be found as connected pixel fields with the sufficient number of pixels. To simplify this search procedure, connected components are tracked on the sequence of frames during the measuring procedure. The initial position as well as the result of the first step are shown in Fig.6. In our case, one video camera pixel corresponds to the area of $0.3 \times 0.3 \mathrm{~mm}$ on the wire surface. Signal-noise ratio is 10:1. The width of the wire section across is equal to $12.8 \mathrm{~mm}$.

In Fig. 6 one can see the wide distribution of laser radiation in vertical direction intensity instead of one thin line. It is supposed that the section along the contact wire length is slightly changing within a large enough distance and the weak image contrast can be taken into account after finding the mass centers $\left(x_{*}, y_{*}\right)$ on the brightness for each a)

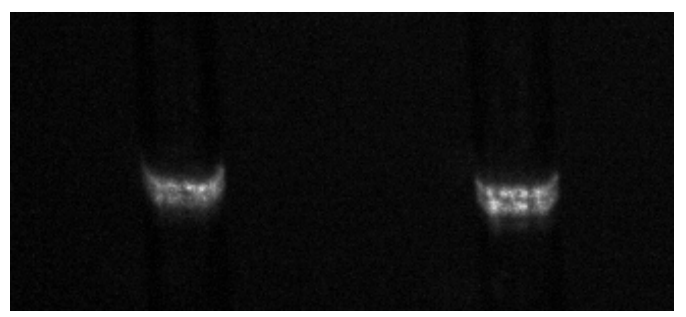

b)

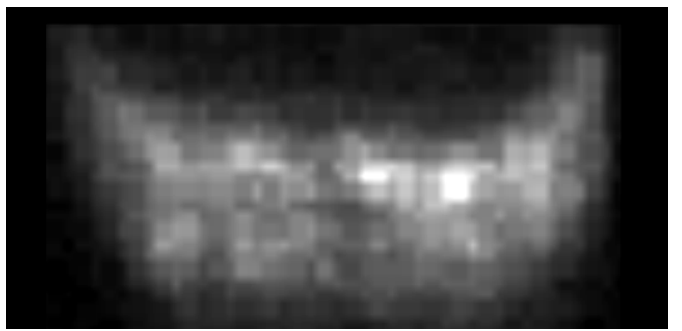

Fig.6 Initial crescent images obtained from video camera (a); marked out and increased crescent image (b)

vertical column of image pixels:

$$
x_{*}=\text { const } ; \quad y_{*}=\frac{\sum_{j} I_{j} y_{j}}{\sum_{j} I_{j}}
$$

as shown in Fig. 7 The summation in the above-mentioned formula is carried out along all pixels with vertical coordinate $y_{j}$ in fixed column of pixels. It is the second step of image analysis algorithms.

After that one can determine the wire section profile under the processing of curve presented by obtained mass centers. For this purpose, using all found centers one can pick up 
algorithmically those centers that belong to unworn-out section of wire. In these fields the wire surface in considered

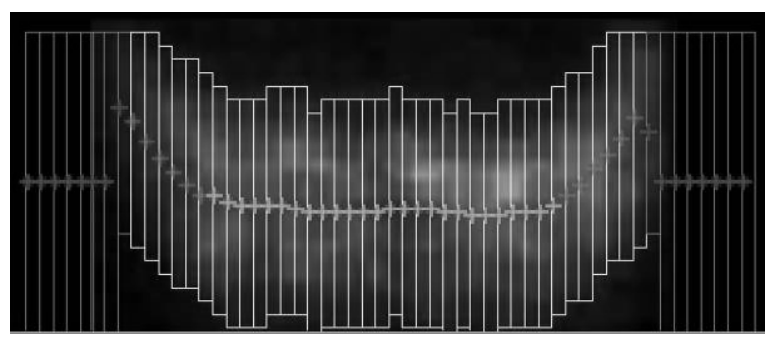

Fig. 7 Crescent image divided into vertical columns with marked out mass center inside each of them

section has a round form that allows using the statistical methods to calculate the center of section contour as a circle center most closely to the initial set of points. As the criteria of closeness, the mean square deviation was chosen.

It is supposed that the section dimension and critical wear height can be determined by a wire mark and given a priori. The center groups and the profile section results are shown in Fig.8.

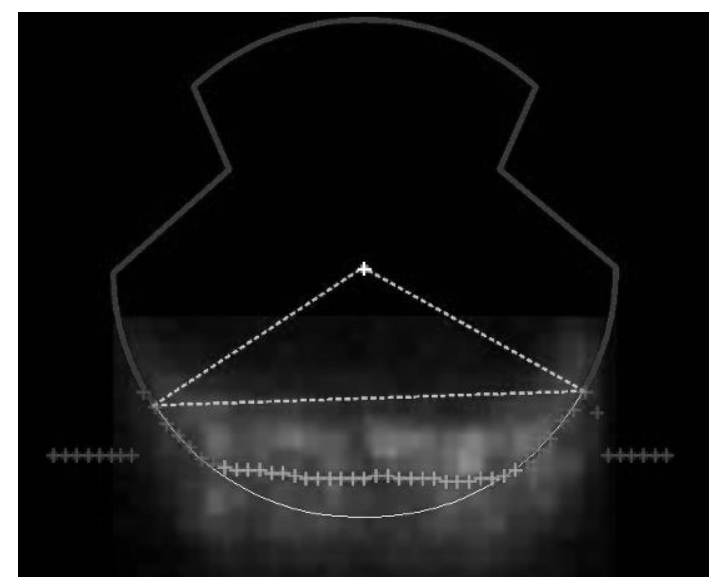

Fig.8 Profile of wire cross-section reconstructed by found center.

Table. Comparison of wire section measurement results by standard method and using the automatic opto-electronic system described in paper

\begin{tabular}{|c|c|c|c|c|c|c|c|}
\hline \multirow{3}{*}{$\begin{array}{l}\text { Measurement } \\
\text { No }\end{array}$} & \multirow{3}{*}{$\begin{array}{l}\text { Rail-Road Pillar } \\
\text { No }\end{array}$} & \multicolumn{2}{|c|}{$\begin{array}{l}\text { Manual wear } \\
\text { measurement, } \\
\text { mm }\end{array}$} & \multicolumn{2}{|c|}{$\begin{array}{c}\text { Optical automatic } \\
\text { wear measurement, } \\
\mathrm{mm}\end{array}$} & \multicolumn{2}{|c|}{$\begin{array}{c}\text { Measurement error, } \\
\mathrm{mm}\end{array}$} \\
\hline & & Left & Right & Left & Right & Left & Right \\
\hline & & wire & wire & wire & wire & wire & wire \\
\hline 1 & 32 & 10.3 & 10.75 & 10.16 & 10.64 & 0.14 & 0.11 \\
\hline 2 & & 10.25 & 10.05 & 10.14 & 10.06 & 0.11 & -0.01 \\
\hline 3 & & 10.3 & 10.05 & 10.21 & 10.07 & 0.09 & -0.02 \\
\hline 4 & 34 & 10.4 & 10.2 & 10.62 & 10.23 & -0.22 & -0.03 \\
\hline 5 & & 10.2 & 10.15 & 10.25 & 10.26 & -0.05 & -0.11 \\
\hline 6 & 36 & 10.35 & 10.6 & 10.19 & 10.55 & 0.16 & 0.05 \\
\hline 7 & & 10.3 & 10.35 & 10.12 & 10.17 & 0.18 & 0.18 \\
\hline 8 & 38 & 10.9 & 10 & 10.72 & 10.21 & 0.18 & -0.21 \\
\hline 9 & & 11.1 & 10.9 & 10.95 & 10.89 & 0.15 & 0.01 \\
\hline \multirow[t]{3}{*}{10} & 40 & 9.85 & 10.5 & 9.88 & 10.49 & -0.03 & 0.01 \\
\hline & & \multicolumn{4}{|c|}{ Mean deviation } & 0.09 & 0.02 \\
\hline & & \multicolumn{2}{|c|}{ RMS deviation } & & & 0.12 & 0.14 \\
\hline
\end{tabular}

In the software used at the video image station, the testing of realized algorithm efficiency is also taken into account. It allows us to determinate the presence of information about the defect under measurement. The images with information about critical wear as well as those thrown away on post criteria are to be put into a special data base together with the processing results.

\section{EXPERIMENTAL TESTING IN THE REAL CONDITIONS}

The measurements that were taken in the real conditions demonstrate that the RMS error of the wear area determination is about $1.5 \mathrm{~mm}^{2}$ at the standard wire cross- sectional area of $100 \mathrm{~mm}^{2}$. In the existing old method of wire's wear measurement, the wire wear is defined by the remaining wire height, which is measured manually with micrometer. The old manual method and new optical one as described here, were compared. The measurements were conducted at train movement speed of $5 \mathrm{~km} / \mathrm{h}$. The results of two methods are compared in the Table.

The results of preliminary system tests demonstrate good agreement of the measured data with the results of direct measurements. The RMS error of the wire height determination did not exceed $0.15 \mathrm{~mm}$. The measurement rate is $\sim 150$ meas./sec., which allows us to provide measurements in $10 \mathrm{~mm}$ steps along the wire length. 
Nowadays the system prototype is under experimental operation at the West-Siberian Railroad.

\section{CONCLUSION}

The optical structured light method and the system industrial prototype for wear measurements and defects detection of a contact wire electro-supply network are presented. Basic advantages of the system are high informativeness and robustness, simultaneous direct crosssection measurement of multiple wires, measurement of acute geometrical defects of wires (like "neck", "rotation", etc), reliable measurements of contact wire parameters at support points (points of maximum wear), determination and analysis of geometrical parameters of wire support structural components, measurement of other important parameters of contact wire network, such as wire "zigzag" and wire "height", as well as the presence of extraneous objects.
The device tested in the real railway conditions extracts main technological wire parameters. First of all, the remaining thickness with the RMS error of the wear area down to $0.15 \mathrm{~mm}$ and also the wear area with the RMS error is about $1.5 \mathrm{~mm}^{2}$ (at the standard wire cross-sectional area of $100 \mathrm{~mm}^{2}$ ). This information is sufficient for a confident decision-making as for a safe operation of the contact wire electro-supply network. This method could be applied for automatic wear measurement and defects detection for trams, trolleybuses and other electro-supply vehicles.

\section{REFERENCES}

[1] Galiulin, Rav. M., Galiulin, Rish. M. et al (2002). Optoelectronic computer aided systems for inspection of gas-turbine engine complex objects. In Proceedings of SPIE 4900: Seventh International Symposium on Laser Metrology Applied to Science, Industry, and Everyday Life, 9-13 September 2002 (pp. 178-184). Washington, USA: SPIE.

[2] Skotheim, O., Couweleers, F. (2004). Structured light projection for accurate $3 \mathrm{D}$ shape determination. In: Proceedings of the 12th International Conference on Experimental Mechanics, August 29 - September 2 2004. Bari, Italy: McGraw-Hill, CD-ROM. 